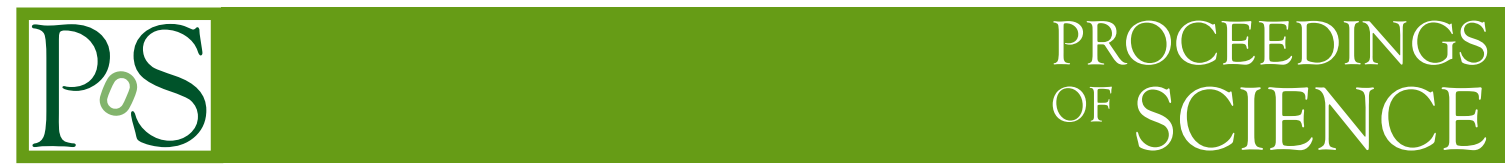

\title{
Is covariant star product unique?
}

\section{Dmitri Vassilevich*}

CMCC - UF ABC (Brazil)

E-mail: dvassilegmail.com

We give a nontechnical introduction to the problem of non-uniqueness of star products and describe a covariant resolution of this problem. Some implications (e.g., for noncommutative gravity) and further prospects are discussed.

Corfu Summer Institute on Elementary Particles and Physics - Workshop on Non Commutative Field Theory and Gravity

September 8 - 12, 2010

Corfu, Greece

${ }^{*}$ Speaker. 


\section{Introduction}

The arena for a noncommutative field theory is a noncommutative manifold, which is defined by replacing the usual point-wise product of functions by a noncommutative star product. This star product depends on a deformation parameter $h$, and the first term in the $h$-expansion is essentially the Poisson bracket of functions. It is a known result by Kontsevich [18] that the Poisson structure defines the star product up to an equivalence. This, however, does not imply that the star product for a given Poisson structure is unique. On the opposite, the family of equivalence transformations is very rich, it depends on an infinite number of arbitrary functions. Consequently, the ambiguity in definition of the star product has an infinite functional dimension. This ambiguity is physically relevant as equivalent star products lead, in general, to inequivalent field theories. Therefore, before going on with constructing a field theory on noncommutative space one has to decide which of the star products has to be used in this construction.

It is natural to request that the algebra equipped with the star product has a number of derivations (represented by covariant derivatives) which satisfy the usual Leibniz rule. However, since derivative of a scalar is a vector, and a product of two vectors is a rank-two tensor, to impose the Leibniz rule one has to extend the star product to all tensor fields. Tensors are characterized by their transformation properties with respect to diffeomorphisms, these properties should be respected by the deformation. Consequently, the star product has to be covariant. This is why we address here the problems of covariance and uniqueness simultaneously.

The paper is organized as follows. In Sec. 2 we introduce star products and describe ambiguities in their definition. Covariant star products are treated in a very short Sec. 3. Our main result [27] is a set of natural conditions which allow to remove the ambiguity almost completely (Sec. 4). Some ambiguity, however, remains. In Sec. 5 we show that it leads to classically equivalent noncommutative field theories. In Sec. 6 we discuss possible extension of the results (to YangMills symmetries, Poisson manifolds, strict deformation quantization) as well as implications for noncommutative gravity.

\section{Non-uniqueness of star products}

In the approach adopted here, which goes back to the seminal papers [3, 4] (see also [8]), the star product is defined through a noncommutative deformation of the algebra $\mathscr{A}$. For simplicity, in this section we suppose that $\mathscr{A}$ is the algebra $C^{\infty}\left(\mathbb{R}^{n}\right)$ of smooth functions on $\mathbb{R}^{n}$. Next, we replace $\mathscr{A}$ by an algebra of formal power series $\mathscr{A}[[h]]$ of the deformation parameter $h$, i.e., of elements of the form

$$
a=a_{0}+h a_{1}+h^{2} a_{2}+\ldots
$$

Here, each $a_{j}$ is a smooth function. A new product is then defined as

$$
a \star b=a \cdot b+\sum_{r=1}^{\infty} h^{r} C_{r}(a, b),
$$

where each $C_{r}$ is a bidifferential operator. This means, for one argument fixed $C_{r}$ is a differential operator acting on the other argument. We stress, that the series (2.1) and (2.2) are formal, i.e., no convergence is assumed. 
In physical applications the deformation parameter $h$ is usually taken to be imaginary.

It is required that the star product is associative, $(a \star b) \star c=a \star(b \star c)$. In the first order of $h$ this yields

$$
C_{1}(a, b)-C_{1}(b, a)=2\{a, b\},
$$

where $\{a, b\}$ is a Poisson bracket,

$$
\{a, b\}=\omega^{\mu v} \partial_{\mu} a \partial_{\nu} b
$$

with some Poisson bivector $\omega^{\mu v}$.

Another common requirement is the Moyal symmetry,

$$
C_{r}(a, b)=(-1)^{r} C_{r}(b, a) .
$$

Then $C_{1}(a, b)$ is defined to be a Poisson bracket. One says that the star product (2.2) is a deformation of the point-wise product in the direction of $\omega^{\mu \nu}$. The problem is to find all star products for a given Poisson structure. Up to second order, a solution may be found by straightforwardly solving the associativity condition,

$$
\begin{aligned}
a \star b=a b+h \omega^{\mu v} \partial_{v} a \partial_{v} b+h^{2}\left(\frac{1}{2} \omega^{\mu v} \omega^{\rho \sigma}\left(\partial_{\mu} \partial_{\rho} a\right)\left(\partial_{v} \partial_{\sigma} b\right)\right. \\
\left.+\frac{1}{3} \omega^{\mu v} \partial_{v} \omega^{\rho \sigma}\left(\left(\partial_{\mu} \partial_{\rho} a\right)\left(\partial_{\sigma} b\right)-\left(\partial_{\rho} a\right)\left(\partial_{\mu} \partial_{\sigma} b\right)\right)\right)+O\left(h^{3}\right) .
\end{aligned}
$$

The existence of a solution to the deformation problem to all orders for an arbitrary Poisson structure was demonstrated by Kontsevich [18], and the solution is not unique. If $\star$ is a product corresponding to some fixed Poisson bivector $\omega^{\mu v}$, then one can define another product $\star^{\prime}$ by

$$
a \star^{\prime} b=D^{-1}(D a \star D b),
$$

where

$$
D=1+h L .
$$

and $L$ being a formal differential operator (a sum of differential operators of arbitrary order with arbitrary polynomial dependence on $h$ ). Clearly, $\star^{\prime}$ is also an associative product corresponding to the same Poisson bivector ${ }^{1}$. The transformation $\star \rightarrow \star^{\prime}$ was called a gauge transformation in [18].

The products $\star$ and $\star^{\prime}$ are equivalent and correspond to isomorphic algebras, but they are different. As we shall see below, field theory models constructed with equivalent star products are in general non-equivalent. Therefore, one has an ambiguity in the choice of the star product corresponding to the ambiguity in the choice of $D$ in (2.7). It is easy to understand that the freedom in $D$ has an infinite functional dimension (infinite number of parameters per point in $\mathbb{R}^{n}$ ).

The situation in noncommutative theories differs drastically from that in, say, general relativity. The metric tensor defines the whole (pseudo-)riemannian structure of the manifold, while to define a star product ${ }^{2}$ (which is the basic structure on a noncommutative space) one has to specify an infinite number of fields contained in $D$ in addition to a Poisson tensor.

\footnotetext{
${ }^{1}$ Actually, Kontsevich [18] demonstrated a more refined statement. One defines a formal Poisson structure, which is, roughly speaking, a Poisson structure which is a formal polynomial in $h$. The classes of equivalence of the star products are in one-to-one correspondence with equivalence classes of formal Poisson structures.

${ }^{2}$ Actual calculation of a star product may also be problematic. The formulae in [19] work till the 5th order in $h$, and beyond this order little is known.
} 


\section{Covariant star products}

The product (2.6) is not covariant at the second order of the expansion in $h$ since it contains usual derivatives of the Poisson tensor. Over last years much progress has been achieved in covariantization of the Kontsevich procedure $[9,1]$ and in extending star products to differential forms [17, 6, 21], to Lie algebra valued differential forms [5], and in covariant holomorphic products [7].

In the symplectic case (non-degenerate $\omega^{\mu v}$ ) situation is much better understood. A covariant star product was suggested already in the seminal paper [3]. An extension of this product to all tensor fields is rather straightforward [26, 27]. The generic construction by Fedosov [10,11] is manifestly covariant.

There is a general feature of (almost) all covariant approaches: to make the star product covariant one needs a connection in addition to the Poisson or symplectic structure.

\section{Reducing the gauge freedom}

From now on we restrict ourselves to symplectic manifolds.

To construct a star product we need some geometric data, which are going to be a symplectic manifold $M$ with a flat symplectic connection $\nabla$. Let $\omega_{\mu \nu}$ be a symplectic form (with the Poisson bivector $\omega^{\mu v}$ being its' inverse, $\omega_{\mu \nu} \omega^{v \rho}=\delta_{\mu}^{\rho}$ ). Let us choose a Christoffel symbol on $M$ such that the symplectic form is covariantly constant,

$$
\nabla_{\mu} \omega_{v \rho}=\partial_{\mu} \omega_{v \rho}-\Gamma_{\mu v}^{\sigma} \omega_{\sigma \rho}-\Gamma_{\mu \rho}^{\sigma} \omega_{v \sigma}=0 .
$$

Therefore, $M$ becomes a Fedosov manifold [14]. Let us suppose that this connection is flat and torsion-free, i.e.,

$$
\left[\nabla_{\mu}, \nabla_{v}\right]=0 .
$$

Locally, one can choose a coordinate system such that $\omega^{\mu v}=$ const . and $\Gamma_{\mu v}^{\sigma}=0$. Such coordinates will be called the Darboux coordinates.

We are going to construct star products for arbitrary tensor fields $\alpha_{n, m} \in T M^{n} \otimes T^{*} M^{m} \equiv T^{n, m}$. This means, $\alpha_{n, m}$ has $n$ contravariant and $m$ covariant indices. Next, we define a covariant Poisson bracket for the tensors

$$
\{\alpha, \beta\}=\omega^{\mu v} \nabla_{\mu} \alpha \cdot \nabla_{v} \beta
$$

possesing all the standard properties of a Possion bracket (antisymmetry, Jacobi identity, etc.). Besides,

$$
\nabla\{\alpha, \beta\}=\{\nabla \alpha, \beta\}+\{\alpha, \nabla \beta\} .
$$

Now, we are ready to construct a star product, which has to be an associative deformation of the point-wise product in the direction of the Poisson bracket (4.4), i.e., it is a product on $T[[h]]$ having the form (2.2) subject to (2.3) (with $a, b$ replaced by $\alpha, \beta$ ). We are interested in covariant products only, meaning that $T^{n_{1}, m_{1}}[[h]] \star T^{n_{2}, m_{2}}[[h]] \subset T^{n_{1}+n_{2}, m_{1}+m_{2}}[[h]]$. Besides, we impose a few "natural" restrictions. Namely, we require stability on covariantly constant tensors

$$
\alpha \star \beta=\alpha \cdot \beta \quad \text { if } \quad \nabla \alpha=0 \text { or } \nabla \beta=0,
$$


the Moyal symmetry

$$
C_{k}(\alpha, \beta)=(-1)^{k} C_{k}(\beta, \alpha)
$$

and that $\nabla$ is a derivation

$$
\nabla \alpha \star \beta=(\nabla \alpha) \star \beta+\alpha \star(\nabla \beta) .
$$

Let us discuss the meaning of (4.5) - (4.7). The first condition (4.5) replaces the usual requirement that the unit element of algebra is not deformed. Physically, it means that slowly varying fields do not see noncommutativity. In applications, the deformation parameter $h$ is imaginary. Therefore, the Moyal symmetry ensures hermiticity of the star product. The last condition (4.7), which nothing else than the Leibniz rule, means that the star product transforms in a controlled way under infinitesimal translations (those generators are the covariant derivatives). In a sense, (4.7) replaces locality of commutative products.

The conditions (4.5) - (4.7) appear to be very restrictive. One can show [27], that if they are satisfied, the star product has the form

$$
\alpha \star_{N} \beta=D^{-1}\left(D \alpha \star_{R} D \beta\right),
$$

where

$$
\alpha \star_{R} \beta=\sum_{k} \frac{h^{k}}{k !} \omega_{R}^{\mu_{1} v_{1}} \ldots \omega_{R}^{\mu_{k} v_{k}}\left(\nabla_{\mu_{1}} \ldots \nabla_{\mu_{k}} \alpha\right) \cdot\left(\nabla_{v_{1}} \ldots \nabla_{v_{k}} \beta\right),
$$

which depends on a "renormalized" symplectic structure

$$
\omega_{R}^{\mu v}=\omega^{\mu v}+h^{2} \omega_{1}^{\mu v}+h^{4} \omega_{2}^{\mu v}+\ldots
$$

with all correction terms $\omega_{j}^{\mu v}$ being covariantly constant,

$$
\nabla_{\rho} \omega_{j}^{\mu v}=0 .
$$

The operator $L$ in the transformation $D$, see (2.8), must have the form

$$
L=\sum_{k=2}^{\infty} L^{\mu_{1} \ldots \mu_{k}} \nabla_{\mu_{1}} \ldots \nabla_{\mu_{k}}
$$

and be a scalar (i.e., $L$ has to be proportional to a unit matrix in the tensor indices) and all $L^{\mu_{1} \ldots \mu_{k}}$ have to be covariantly constant,

$$
\nabla_{v} L^{\mu_{1} \ldots \mu_{n}}=0,
$$

and may contain odd powers of the deformation parameter only.

The product (4.9) is nothing else than a covariantization of the Moyal product [3] extended to tensors [26, 27].

The proof [27] of this statement is rather hard and technical, but we shall try to give here a rough idea how does it go. First, let us note that the product (4.8) with an arbitrary form differential operator $D$ is a deformation of the pointwise product of tensors in the direction of the Poisson brackets (4.3). Therefore, our task is to analyze restrictions on $D$. The "gauge freedom" contained in $D$ is much larger than in the scalar case. Besides being a differential operator, $L$ may be a complicated linear transformation of tensors. Mixing up tensors of different degree is forbidden 
by the covariance. Therefore, $L^{\mu_{1} \ldots \mu_{n}}=\oplus_{n, m} L_{n, m}^{\mu_{1} \ldots \mu_{n}}$, where $L_{n, m}^{\mu_{1} \ldots \mu_{n}}$ is a restriction to the tensors of degree $(n, m)$. Next, by using (4.5), one can show that $L_{n, m}^{\mu_{1} \ldots \mu_{n}}=I_{(n, m)} L_{0,0}^{\mu_{1} \ldots \mu_{n}}$, where $I_{(n, m)}$ is the identity map. Further restrictions are then obtained by analyzing the Leibniz rule (4.7). The Moyal symmetry (4.6) is not very essential, though useful to remove certain powers of the deformation parameter, as, e.g., odd powers of $h$ in (4.10).

The ambiguity in the star products has been reduced enormously. Instead of an infinity number of fields in the gauge operator $D$ we have just an infinite number of constants. Nevertheless, some freedom still remains, and we like to discuss its' meaning. The freedom encoded in (4.10) is rather harmless. This is the price to pay for working in formal setup. In physical application the series in (4.10) must be summed up somehow. Only the "renormalized" value $\omega_{R}$ is of relevance, while the way how we split it in the perturbation series is no more than a technical device. The rest of the gauge freedom is discussed in the next section.

\section{Classical field theory}

To understand consequences of the freedom encoded in remaining gauge transformations one has consider the integration. There is a natural measure on symplectic manifolds [12]

$$
d \mu(x)=\left(\operatorname{det}\left(\omega^{\mu v}\right)\right)^{-\frac{1}{2}} d x .
$$

With respect to this measure, the star product $\star_{R}$ is closed, i.e.,

$$
\int_{M} d \mu(x) \alpha_{\mu \nu \ldots \rho} \star \beta^{\mu \nu \ldots \rho}=\int_{M} d \mu(x) \alpha_{\mu \nu \ldots \rho} \cdot \beta^{\mu \nu \ldots \rho} .
$$

provided all indices are contracted in pairs (or, equivalently, if the integrand is diffeomorphism invariant). Among the products $\star_{N}$ not all are closed.

Consider an action of a classical field theory on noncommutative space equipped with the product $\star_{N}$

$$
S=\int d \mu(x) P\left(f_{i}, \nabla\right)_{\star_{N}},
$$

where $f_{i}$ are some fields, $P$ is a polynomial, where all products are $\star_{N}$ products. We can rewrite $S$ as

$$
S=\int d \mu(x) D^{-1}\left(P\left(D f_{i}, \nabla\right)_{\star_{R}}\right)=\int d \mu(x) P\left(D f_{i}, \nabla\right)_{\star_{R}} .
$$

This means, that the replacement $\star_{N}$ by $\star_{R}$ is compensated by the transformation $f_{i} \rightarrow D f_{i}$. Since the operator $D$ is invertible, the theories based on the two star products are classically equivalent. Therefore, the remaining freedom is also harmless. For physical applications, the star products related by gauge transformations with covariantly constant coefficients in $L$ are equivalent.

The same calculations also shows why the theories based on gauge-equivalent star products are, in general, non equivalent. If the tensors $L^{\mu_{1} \ldots \mu_{k}}$ are not all covariantly constant, one cannot delete the operator $D^{-1}$ is (5.4). 


\section{What is next?}

\subsection{Yang-Mills gauge covariance}

For physical applications is important to have a star product which is covariant with respect to the Yang-Mills gauge transformation. A product of this type was constructed in [25] by introducing a flat (gauge-trivial) connection and covariantization of the Moyal product. That paper did not consider the problem of uniqueness of gauge-covariant star products, and here we briefly comment on how this can be achieved. First of all, introducing a matter field belonging to certain representation of the gauge group requires an extension of the space of function on which the star product is defined to a direct sum of all representations appearing in tensor powers of the initial one. This is similar to introducing the spaces $T^{n, m}$, see above. Then, one has to impose a set of "natural" restrictions on the star product, with most important ones being the stability on covariantly constant fields, the Leibniz rule, and the Moyal symmetry (exactly in parallel to Eqs. (4.5) - (4.7)). Then one observes that the Moyal-type product (4.9) with the gauge connection introduced above satisfies all the requirements. Therefore, the problem is to find the restrictions of the Kontsevich transformations $D$ which also respect that requirements. The calculations go like in the previous case, but are much simpler as the gauge connection does not change the representation of the gauge to which the field belongs (in contrast to the tensor degree). It is not surprising therefore that the final result looks precisely as before with just the connection being suitably modified.

\subsection{Poisson manifolds}

On the physical grounds it is hard to explain why $\omega^{\mu v}$ has to be non-degenerate. Therefore, one has to be able to work in a more general setting of Poisson manifolds. This case is much more complicated than the symplectic one, as, for example, no Moyal-type formula (see (4.9) exists. This is related to non-existence of a connection such that $\nabla \omega^{\mu v}=0$ since covariant constancy of $\omega$ implies that its' rank is a constant, which is not always true on Poisson manifolds. This can be partially corrected by introducing the so-called contravariant connection [24]. On the other hand, the construction of the universal star product [1] starts with an arbitrary connection, and one can be quite optimistic about a possibility to extend this construction to tensors and to study the consequence of the Leibniz rule and other restrictions which we imposed above in the symplectic case. As we have already explained above, a honest implementation of the Leibniz rule requires an extension to tensors.

\subsection{Beyond formality}

In physical applications it is desirable to sum up the series in deformation parameter to obtain a star product between functions on a manifold rather than between formal power series. One has to be able to give a numerical value to $h$, and to make sense of the notions like "the characteristic scale of noncommutativity". From mathematical point of view, summing up formal series is a step towards using the full power of noncommutative geometry [15]. The problem of going beyond formality is extremely complicated. There are just a few manifolds which are noncommutative in the strict sense. Therefore, it is probably more reasonable to restrict ourselves to construcing new examples. The existence of a flat torsionless symplectic connection (and of some more useful 
structures) is guaranteed on rigid special Kähler manifolds [13, 20, 2] which seem to be ideal candidates for (strict) noncommutative deformations ${ }^{3}$.

\subsection{Towards noncommutative gravity}

Proper realization of the diffeomorphism invariance is one of the most important issues for constructing noncommutative gravity theories [23]. Of course, having a diffeomorphism covariant star product helps a lot. In particular, one can construct [26] noncommutative counterparts for all dilaton gravities in two dimension [16] having a full diffeomorphism invariance group, which by itself looks as a rather strong result. Since one of the models appeared to be integrable to all orders of the noncommutativity parameter, analyzing the solutions allows to highlight some general problems arising in noncommutative gravity models. The dilaton gravity models in the first order formulation contain a zweibein which can be represented through a complex one-form $e_{\mu}$ so that the Lorentz transformations become multiplications with a local phase factor. In NC models this multiplications becomes a star multiplication, which can be fixed to be a left star multiplication

$$
\delta e_{\mu}=i \lambda \star e_{\mu}, \quad \delta \bar{e}_{\mu}=-i \bar{e}_{\mu} \star \lambda,
$$

where $\lambda$ is a parameter. The transformation of complex conjugate zweibein $\bar{e}_{\mu}$ involves right star multiplication. (This follows from the Moyal symmetry and the fact that $h$ has to be considered imaginary). There is then a unique metric

$$
g_{\mu v}=\frac{1}{2}\left(\bar{e}_{\mu} \star e_{v}+\bar{e}_{\nu} \star e_{\mu}\right),
$$

which is real, symmetric, invariant with respect to (6.1) and does not contain derivatives. (The derivatives inside the star product do not count). Since the star product is diffeomorphism covariant, the metric (6.2) defines a diffeomorphism invariant line element. Dilator gravity models also contain a dilaton, a connection one-form, and two auxiliary fields which generate torsion constraints. None of them will be important below. It is not hard to construct a noncommutative 2D dilaton gravity that is integrable and admits gauge-trivial solutions only [26]. In other words, up to a Lorentz transformation,

$$
e_{\mu}=\partial_{\mu} E
$$

where $E$ is a complex scalar function. By a suitable choice of the coordinate, taking $E=x^{1}+$ $i x^{2}$, the zweibein can be made the unit matrix. On the other hand, there is a coordinate system where the symplectic structure is constant and the connection is trivial. In such a system the star product becomes just the usual Moyal product. The Moyal product of two unit zweibeins is a unit metric, and one may conclude that the model describes a flat space geometry, as expected. The last statement is however wrong as the zweibein and the symplectic structure trivialize in two different coordinate systems, in general. To understand the consequences, let us consider an example. As we have just mentioned above, one can choose a coordinate system to simplify the symplectic structure and to make the star product the usual Moyal product,

$$
\alpha \star \beta=\exp \left(\left.i \theta\left(\partial_{1}^{x} \partial_{2}^{y}-\partial_{2}^{x} \partial_{1}^{y}\right) \alpha(x) \beta(y)\right|_{y=x} .\right.
$$

\footnotetext{
${ }^{3}$ I am grateful to Paolo Aschieri for drawing my attention to special Kähler geometries.
} 
Let us take the function $E$ in most simple but yet nontrivial form, $E=\sin \left(x^{1}\right)+i \sin \left(x^{2}\right)$. Calculating the metric and corresponding invariants is an easy exercise, (one can also look up in[27]), showing that in this example we are dealing with a very non-trivial geometry. The metric even changes signature at some values of $x^{1}$ and $x^{2}$. The lesson one can learn from this example is not that noncommutative gravity predicts signature changes, but rather that the metric can behave in a very wild way. To avoid this, one needs to impose a relation between the metric and the symplectic structure. Interesting relations of that kind follow from the matrix models [22].

Same problem exists in most of the approaches to noncommutative gravity, specifically in that based on a fixed star product. Many such models are presented in the review paper by Szabo [23]. For each of these models one should either demonstrate that noncommutative corrections to a classical geometry (say, Schwarzschild black hole) are physically equivalent independently in which particular coordinate system (e.g., Schwarzschild or Eddington-Finkelstein) the star product is Moyal, or to propose a principle which relates the metric to the star product.

\section{Acknowledgement}

I am grateful to Dorothea Bahns, Harald Grosse and George Zoupanos for organizing such a wonderful workshop, for their support and stimulating atmosphere. This work was supported in part by FAPESP and CNPq.

\section{References}

[1] M. Ammar, V. Chloup and S. Gutt, Universal star products, Lett. Math. Phys. 84 (2008) 199 [arXiv:0804.1300[math.SG]].

[2] P. Aschieri, S. Ferrara and B. Zumino, Duality Rotations in Nonlinear Electrodynamics and in Extended Supergravity, Riv. Nuovo Cim. 31 (2008) 625 [arXiv: 0807.4039 [hep-th]].

[3] F. Bayen, M. Flato, C. Fronsdal, A. Lichnerowicz and D. Sternheimer, Deformation Theory and Quantization. 1. Deformations of Symplectic Structures, Annals Phys. 111 (1978) 61.

[4] F. Bayen, M. Flato, C. Fronsdal, A. Lichnerowicz and D. Sternheimer, Deformation Theory and Quantization. 2. Physical Applications, Annals Phys. 111 (1978) 111.

[5] M. Chaichian, M. Oksanen, A. Tureanu and G. Zet, Noncommutative gauge theory using covariant star product defined between Lie-valued differential forms, Phys. Rev. D 81 (2010) 085026 [arXiv:1001.0508 [hep-th]].

[6] M. Chaichian, M. Oksanen, A. Tureanu and G. Zet, Covariant star product on symplectic and Poisson spacetime manifolds, Int. J. Mod. Phys. A 25 (2010) 3765 [arXiv:1001.0503 [math-ph]].

[7] L. Cornalba, Matrix representations of holomorphic curves on T(4), JHEP 0008 (2000) 047 [arXiv:hep-th/9812184].

[8] G. Dito and D. Sternheimer, Deformation Quantization: Genesis, Developments and Metamorphoses, IRMA Lect. Math. Theor. Phys. 1, de Gruyter, Berlin, p.9 (2002) [arXiv:math/0201168].

[9] V. Dolgushev, Covariant and equivariant formality theorem, Adv. Math. 191 (2005) 147.

[10] B. Fedosov, Formal quantization in Some Topics of Modern. Math. and Their Appl. to Problems of Math. Phys, ed. L. D. Kudryavtsev, Moscow, Phys. Tech. Inst. (1985) 129 
[11] B. Fedosov, A Simple Geometrical Construction of Deformation Quantization, J. Diff. Geom. 40 (1994) 213.

[12] G. Felder and B. Shoikhet, Deformation quantization with traces, Lett. Math. Phys. 53 (2000), 75-86 [arXiv:math/0002057].

[13] D. S. Freed, Special Kaehler manifolds, Commun. Math. Phys. 203 (1999) 31 [arXiv:hep-th/9712042].

[14] I. Gelfand, V. Retakh and M. Shubin, Fedosov Manifolds, Adv. Math. 136 (1998) 104.

[15] J. M. Gracia-Bondía, J. C. Várily and H. Figueroa, Elements of Noncommutative Geometry, Birkhäuser, Boston, 2001.

[16] D. Grumiller, W. Kummer and D. V. Vassilevich, Dilaton gravity in two dimensions, Phys. Rept. 369 (2002) 327 [arXiv:hep-th/0204253].

[17] P. M. Ho and S. P. Miao, Noncommutative differential calculus for D-brane in non-constant B field background, Phys. Rev. D 64 (2001) 126002 [arXiv: hep-th/0105191].

[18] M. Kontsevich, Deformation quantization of Poisson manifolds, I, Lett. Math. Phys. 66 (2003) 157 [arXiv:q-alg/9709040].

[19] V. G. Kupriyanov and D. V. Vassilevich, Star products made (somewhat) easier, Eur. Phys. J. C 58, 627 (2008) [arXiv:0806.4615 [hep-th] ].

[20] M. A. Lledo, O. Macia, A. Van Proeyen and V. S. Varadarajan, Special geometry for arbitrary signatures, arXiv:hep-th/0612210.

[21] S. McCurdy and B. Zumino, Covariant Star Product for Exterior Differential Forms on Symplectic Manifolds, AIP Conf. Proc. 1200 (2010) 204 [arXiv:0910.0459 [hep-th] ].

[22] H. Steinacker, Emergent Geometry and Gravity from Matrix Models: an Introduction, Class. Quant. Grav. 27 (2010) 133001 [arXiv: 1003.4134 [hep-th] ].

[23] R. J. Szabo, Symmetry, gravity and noncommutativity, Class. Quant. Grav. 23 (2006) R199 [arXiv:hep-th/0606233].

[24] I. Vaisman, On the geometric quantization of Poisson manifolds, J. Math. Phys. 32 (1991) 3339.

[25] D. V. Vassilevich, Symmetries in noncommutative field theories: Hopf versus Lie, São Paulo J. Math. Sci. 4 (2010) 121-133 [arXiv:0711.4091 [hep-th] ].

[26] D. V. Vassilevich, Diffeomorphism covariant star products and noncommutative gravity, Class. Quant. Grav. 26 (2009) 145010 [arXiv:0904.3079 [hep-th] ].

[27] D. V. Vassilevich, Tensor calculus on noncommutative spaces, Class. Quant. Grav. 27 (2010) 095020 [arXiv:1001.0766 [hep-th]]. 